\title{
Effects of Ga Substitution on Crystallographic and Magnetic Properties of Co Ferrites
}

\author{
Kwang Pyo Chae ${ }^{1 *}$, Won-Ok Choi ${ }^{1}$, Byung-Sub Kang ${ }^{1}$, and Young Bae Lee ${ }^{2}$ \\ ${ }^{1}$ Department of Nano Science and Mechanical Engineering, Nanotechnology Research Center, Konkuk University, \\ Chungiu 380-701, Korea \\ ${ }^{2}$ Department of Physics, Hanzhong University, Donghae 240-713, Korea
}

(Received 11 November 2014, Received in final form 17 December 2014, Accepted 30 December 2014)

\begin{abstract}
The crystallographic and magnetic properties of gallium-substituted cobalt ferrite $\left(\mathrm{CoGa}_{\mathbf{x}} \mathrm{Fe}_{2-\mathrm{x}} \mathrm{O}_{4}\right)$ were investigated. The new material was synthesized using conventional ceramic methods, with gallium substituted for ferrite in the range of $x=0.0$ to 1.0, in steps of 0.2. X-ray diffraction and Mössbauer spectroscopy were used to confirm the presence of crystallized particles in the $\mathrm{CoGa}_{\mathrm{x}} \mathrm{Fe}_{2-\mathrm{x}} \mathrm{O}_{4}$ ferrite powders. All of the samples exhibited a single phase with a spinel structure, and the lattice parameters decreased as the gallium content increased. The particle size of the samples also decreased as gallium increased. For $x \leq 0.4$, the Mössbauer spectra of $\mathrm{CoGa}_{\mathrm{x}} \mathrm{Fe}_{2-\mathrm{x}} \mathrm{O}_{4}$ could be fitted with two Zeeman sextets, which are the typical spinel ferrite spectra of $\mathrm{Fe}^{3+}$ with A- and B-sites. However, for $\mathrm{x} \geq 0.6$, the Mössbauer spectra could be fitted with two Zeeman sextets and one doublet. The variation in the Mössbauer parameters and the absorption area ratio indicated a cation distribution of $\left(\mathrm{Co}_{0.2-0.2 \mathrm{x}} \mathrm{Ga}_{\mathrm{x}} \mathrm{Fe}_{0.8-0.6 \mathrm{x}}\right)\left[\mathrm{Co}_{0.8+0.2 \mathrm{x}} \mathrm{Fe}_{1.2-0.4 \mathrm{x}}\right] \mathrm{O}_{4}$, and the magnetic behavior of the samples suggested that the increase in gallium content led to a decrease in the saturation magnetization and in the coercivity.
\end{abstract}

Keywords : cobalt gallium ferrite, Mössbauer spectroscopy, cation distribution, saturation magnetization, coercivity

\section{Introduction}

Cobalt ferrite, $\mathrm{CoFe}_{2} \mathrm{O}_{4}$, is a hard magnetic material with a high coercivity, moderate saturation magnetization, and remarkable chemical stability and mechanical hardness $[1,2]$. Cobalt ferrite basically has an inverse spinel structure with a corrected cation distribution of $\left(\mathrm{Co}_{0.1} \mathrm{Fe}_{0.9}\right)$ $\left[\mathrm{Co}_{0.9} \mathrm{Fe}_{1.1}\right] \mathrm{O}_{4}$, and the degree of inversion depends on the thermal history of the sample.

This material has been extensively studied, and the substitution of nonmagnetic and diamagnetic ions in spinel ferrite has been observed to affect the magnetic hyperfine fields. Gallium substitution into ferrite has an interesting effect on the electric and magnetic properties. For example, as the Ga content in NiCuZn ferrite increases, the magnetic hyperfine field, the conductivity, and the transition temperature all decrease [3]. In Ga-substituted Ni ferrite, the Ni ion inversion is affected by the quantity of Ga ions that are present, and the dielectric constant and the active energy increase with an increase in the $\mathrm{Ga}$

(C)The Korean Magnetics Society. All rights reserved.

*Corresponding author: Tel: +82-43-840-3623

Fax: +82-43-851-4169, e-mail: kpchae@kku.ac.kr content [4]. The effects that Ga substitution has on the magnetic hyperfine field of $\mathrm{Mn}$ ferrite have been qualitatively explained according to the supertransferred hyperfine interaction [5]. To the best of our knowledge, however, few studies have detailed the crystallographic and magnetic properties of gallium-substituted cobalt ferrite. In order to do so, we synthesized $\mathrm{CoGa}_{\mathrm{x}} \mathrm{Fe}_{2-\mathrm{x}} \mathrm{O}_{4}$ ferrites and studied their crystallographic and magnetic properties by using Xray diffractometry (XRD), scanning electron microscopy (SEM), Mössbauer spectroscopy, and vibrating sample magnetometry (VSM).

\section{Experiment}

The gallium-substituted cobalt ferrite powders, $\mathrm{CoGa}_{\mathrm{x}}-$ $\mathrm{Fe}_{2-\mathrm{x}} \mathrm{O}_{4}$, were prepared with a gallium concentration ranging from $\mathrm{x}=0.0$ to 1.0 , with step of 0.2 using a conventional ceramic method. $\mathrm{Co}_{2} \mathrm{O}, \mathrm{Ga}_{2} \mathrm{O}_{3}$, and $\mathrm{Fe}_{2} \mathrm{O}_{3}$ powders were all $99.99 \%$ pure. After the powders were dried at $100^{\circ} \mathrm{C}$ for $2 \mathrm{~h}$, the mixtures were milled for $1 \mathrm{~h}$ and were pressed into a pellet form using a hydraulic press at 5 ton/ $\mathrm{cm}^{2}$. The pellets were then sintered in a furnace, annealed at $900^{\circ} \mathrm{C}$ for $12 \mathrm{~h}$, milled once again, pressed into a pellet form, and annealed at $1100^{\circ} \mathrm{C}$ for $12 \mathrm{~h}$. These samples 
were used in a powder form during the X-ray analysis and the Mössbauer experiment. The X-ray diffraction (Philips X'pert-MPD) patterns of the powder samples were obtained at room temperature with $\mathrm{Cu} \mathrm{k \alpha}(\lambda=$ $1.5418 \AA$ ) radiation. The surface of the microstructure was observed via FESEM (Hitachi 3000) at room temperature, and the Mössbauer spectra were obtained with a conventional electromechanical type Mössbauer spectrometer (FAST Com. Tec.) with a ${ }^{57} \mathrm{Co}$ source (10 $\mathrm{mCi}$ ) in a rhodium matrix. The magnetic properties were measured using a VSM (Lake Shore 7404).

\section{Results and Discussion}

Fig. 1 shows the X-ray diffraction patterns of galliumsubstituted cobalt ferrite, $\mathrm{CoGa}_{\mathrm{x}} \mathrm{Fe}_{2-\mathrm{x}} \mathrm{O}_{4}$, with $0.0 \leq \mathrm{x} \leq$ 1.0. The X-ray diffraction peaks for these samples indicate that the material has a typical spinel structure with an $\mathrm{Fd} 3 \mathrm{~m}$ space group that also suggests the absence of any secondary phase. The value of the lattice constant $a$ was calculated using the miller index of JCPDS card compare with the measured $d$ values and the Bragg angles. More accurate lattice constant obtained from the least square fitting using Nelson-Riley function. The lattice constant decreased within an acceptable range with an experimental error of $0.839 \mathrm{~nm}$ for $\mathrm{x}=0$ and $0.836 \mathrm{~nm}$ for $\mathrm{x}=$ 1.0, as shown in Fig. 2(a). The observations were consistent with Vegard's law [6], which provides an explanation of the linear variation in the lattice constant

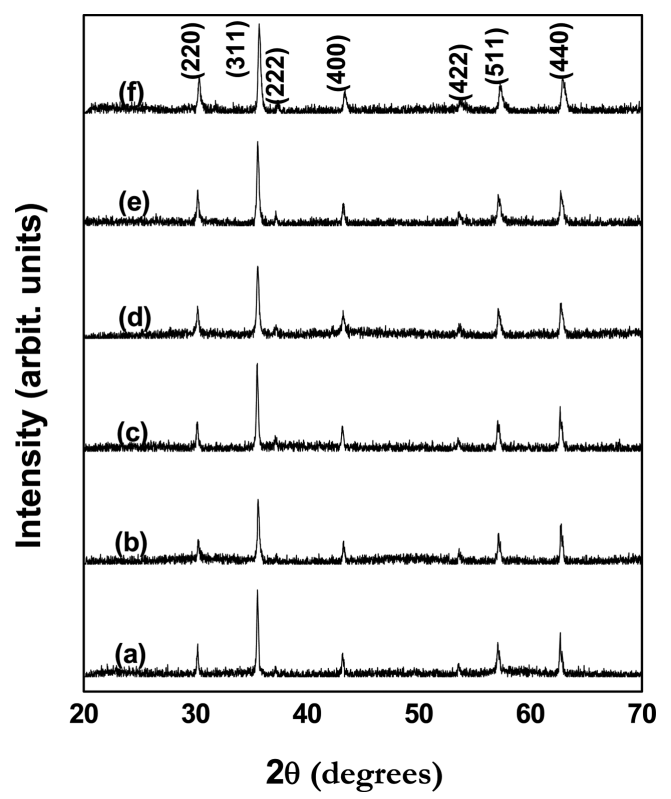

Fig. 1. X-ray diffraction patterns of $\mathrm{CoGa}_{\mathrm{x}} \mathrm{Fe}_{2-\mathrm{x}} \mathrm{O}_{4}$ ferrites: (a) $\mathrm{x}=0.0$, (b) $\mathrm{x}=0.2$, (c) $\mathrm{x}=0.4$, (d) $\mathrm{x}=0.6$, (e) $\mathrm{x}=0.8$ and (f) $\mathrm{x}=1.0$.

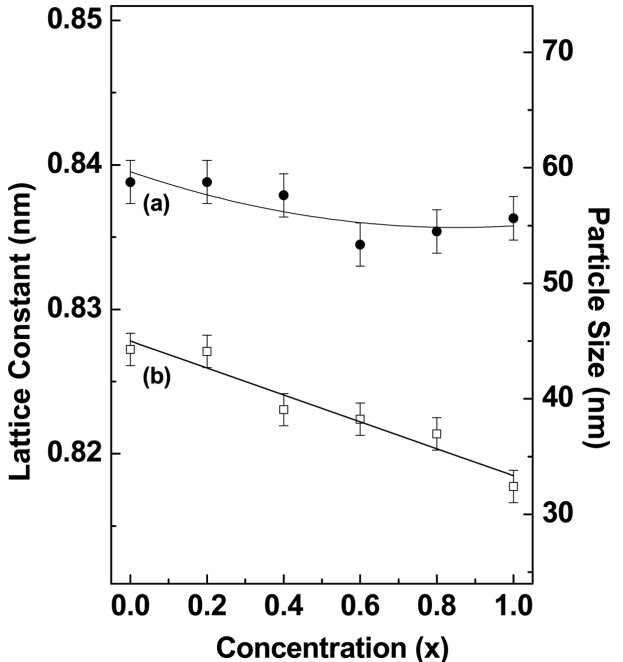

Fig. 2. Variation of the lattice constant and the particle sizes of $\mathrm{CoGa}_{\mathrm{x}} \mathrm{Fe}_{2-\mathrm{x}} \mathrm{O}_{4}$ ferrites.

with an increase in the ionic radii of the doped and the replacing ion. In this study, we replaced $\mathrm{Fe}^{3+}$ ions with $\mathrm{Ga}^{3+}$ ions, and the ionic radius of $\mathrm{Ga}^{3+}$ ions is smaller at $0.62 \AA$ than that of $\mathrm{Fe}^{3+}$ ions at $0.64 \AA$. Thus, replacing $\mathrm{Fe}^{3+}$ ions with $\mathrm{Ga}^{3+}$ ions results in a decrease in the lattice constant of ferrite. The size of the particles was determined by measuring the diffraction peak broadening and using the Scherrer equation [7] $\left.t=(0.9 \lambda) / B \cos \theta_{B}\right)$, where $\lambda$ represents the X-ray wavelength, $B$ is the half width of the (311) peak, and $\theta_{B}$ is the angle of the (311) peak. The particle size of the $\mathrm{CoGa}_{\mathrm{x}} \mathrm{Fe}_{2-\mathrm{x}} \mathrm{O}_{4}$ ferrite powders linearly decreased within the acceptable range with an experimental error of $44.3 \mathrm{~nm}$ for $\mathrm{x}=0$ and $32.4 \mathrm{~nm}$ for $\mathrm{x}=1.0$, as shown in Fig. 2(b). It is known that the ferrite powders that are prepared by using the ceramic method exhibit a non-uniform grain size distribution with the grain size greater than several $\mu \mathrm{m}$. Figure 3 shows the SEM images of the $\mathrm{CoGa}_{\mathrm{x}} \mathrm{Fe}_{2-\mathrm{x}} \mathrm{O}_{4}$ ferrite powders. The photomicrographs were taken with a magnification of 20,000 times and at the same scale for the sample. As the gallium concentration increases, some of the smaller grains become embedded among larger grains, and these grains increase in number.

The X-ray results show that a small gallium substitution leads to no other phase in the spinel structure. However, the Mössbauer spectrum provides a means of studying the microstructural properties of the substituted systems in a way in which the X-ray diffraction and the magnetic susceptibility measurements cannot, and it is, therefore, of practical value in this study. The Mössbauer absorption spectra measured at room temperature for $\mathrm{CoGa}_{\mathrm{x}} \mathrm{Fe}_{2-\mathrm{x}} \mathrm{O}_{4}$ are shown in Fig. 4. The spectra of the $\mathrm{x} \leq 0.4$ samples 


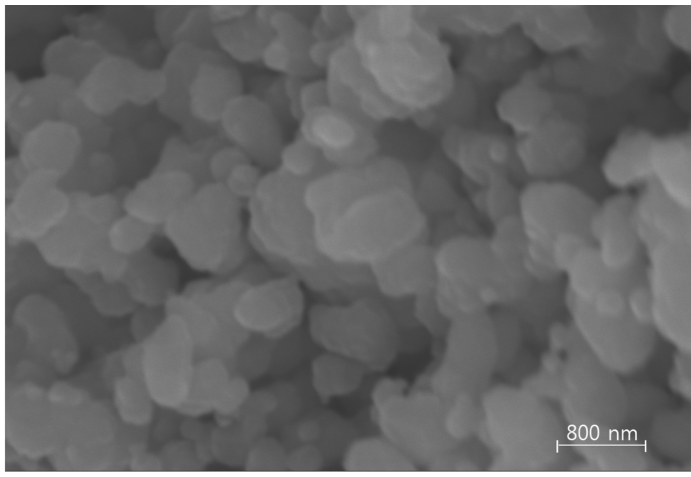

(a)

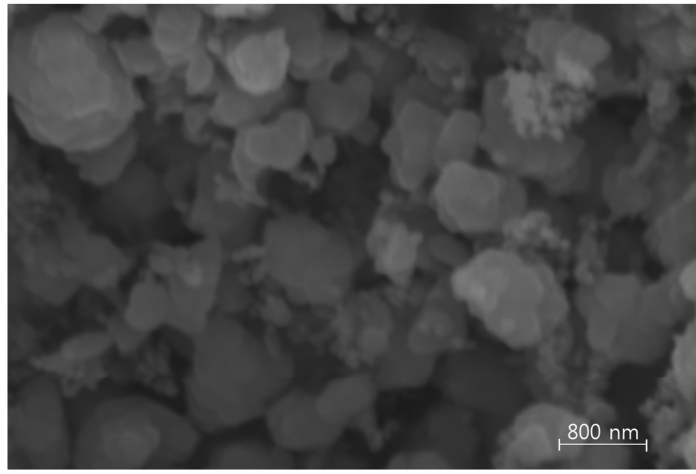

(b)

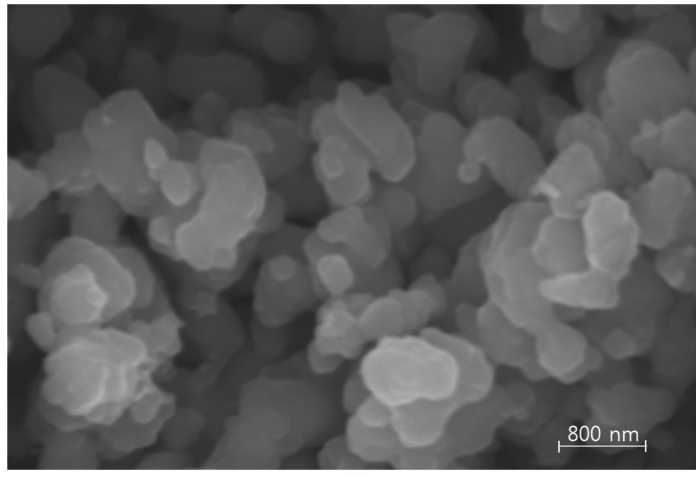

(c)

Fig. 3. SEM images of $\mathrm{CoGa}_{\mathrm{x}} \mathrm{Fe}_{2-\mathrm{x}} \mathrm{O}_{4}$ ferrites: (a) $\mathrm{x}=0.0$, (b) $\mathrm{x}=0.6$ and $(\mathrm{c}) \mathrm{x}=1.0$.

are fitted with two sextets subspectra, which were assigned to the $\mathrm{Fe}^{3+}$ at the tetrahedral A-site and the octahedral B-site of the typical spinel crystal structure. However, the spectra of the $x \geq 0.6$ samples are fitted with two sextets and one doublet, as shown in Fig. 4 and Table 1. The isomer shift $(I S)$ values at room temperature are 0.28-0.37 $\mathrm{mm} / \mathrm{s}$ relative to the $\mathrm{Fe}$ metal, which are consistent with high-spin $\mathrm{Fe}^{3+}$ charge state. The quadrupole splitting $(Q S)$ values for both the A- and the B-sites of the Zeeman sextets are almost 0 , which is in accordance with their cubic crystal structure. The Fe ions that do not participate in the superexchange process in the spinel structure will

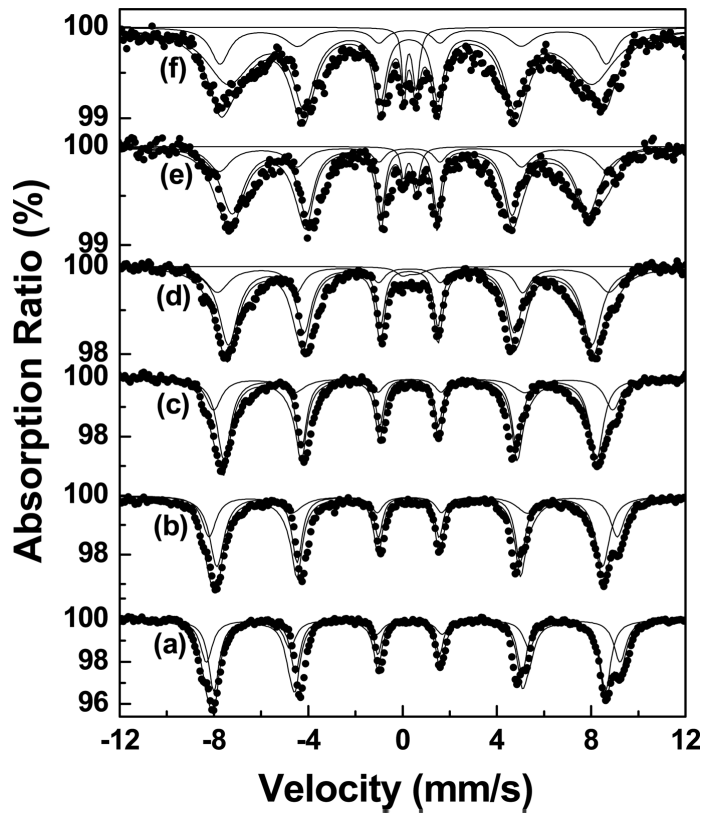

Fig. 4. Mössbauer spectra at room temperature of $\mathrm{CoGa}_{x} \mathrm{Fe}_{2}$ ${ }_{\mathrm{x}} \mathrm{O}_{4}$ ferrites: (a) $\mathrm{x}=0.0$, (b) $\mathrm{x}=0.2$, (c) $\mathrm{x}=0.4$, (d) $\mathrm{x}=0.6$, (e) $\mathrm{x}=0.8$ and (f) $\mathrm{x}=1.0$.

show doublet in the Mössbauer spectrum, and similar results have been observed in aluminum-substituted LiCo-Ti ferrites [8].

As shown in Table 1, in the Zeeman sextets, the isomer shift and the quadruple splitting values are nearly constant with the substituted gallium contents. However, the Mössbauer parameters show a decrease in the $H_{h f}$ value as the content of the substituted gallium increases. This indicates that the substitution of the nonmagnetic gallium ions has an influence on the superexchange interaction in the A- and the B-sites in the spinel structure. In particular, the $H_{h f}$ of the B-site more rapid decreases than that of the A-site. The doublet area of the Mössbauer spectra increases as the substituted gallium content increases, and the areas of the A- and the B-sites exhibit some reduction as the gallium concentration increases, indicating that the substituted gallium ions mostly enter into the spinel sites, substituting for the Fe ions.

The cation distribution can be determined from the site preference and the Mössbauer spectra ratio. The cation distribution depends on many factors, including the temperature, pressure, and composition, as well as on the compound preparation method. In Fig. 4, the areas of the B-sites in the Zeeman sextets exhibit small decreases whereas those of the A-sites show rapid decrease as the concentration of the substituted gallium increases. This indicates that the gallium ions enter into the A-site and substitute for the iron ions. The cobalt ions that enter into 
Table 1. Room-temperature Mössbauer parameters of the gallium-substituted cobalt ferrite, $\mathrm{CoGa}_{\mathrm{x}} \mathrm{Fe}_{2-\mathrm{x}} \mathrm{O}_{4}$. $H_{h f}$ represents the magnetic hyperfine field, $Q S$ represents the quadrupole splitting, and $I S$ represents the isomer shift relative to metallic iron.

\begin{tabular}{|c|c|c|c|c|c|c|c|}
\hline \multirow{2}{*}{$\mathrm{x}$} & \multirow{2}{*}{ spectrum } & \multicolumn{2}{|c|}{$H_{h f}(\mathrm{kOe})$} & \multicolumn{2}{|c|}{$Q S(\mathrm{~mm} / \mathrm{s})$} & \multicolumn{2}{|c|}{$I S(\mathrm{~mm} / \mathrm{s})$} \\
\hline & & B-site & A-site & B-site & A-site & B-site & A-site \\
\hline 0.0 & sextet & 515.0 & 544.0 & 0.02 & 0.08 & 0.28 & 0.37 \\
\hline 0.2 & sextet & 507.0 & 537.0 & 0.02 & 0.08 & 0.29 & 0.37 \\
\hline 0.4 & sextet & 490.0 & 525.0 & 0.02 & 0.08 & 0.30 & 0.37 \\
\hline \multirow{2}{*}{0.6} & sextet & 478.0 & 515.0 & 0.02 & 0.08 & 0.30 & 0.37 \\
\hline & doublet & \multicolumn{2}{|c|}{-} & \multicolumn{2}{|c|}{0.60} & \multicolumn{2}{|c|}{0.30} \\
\hline \multirow{2}{*}{0.8} & sextet & 468.0 & 510.0 & 0.02 & 0.08 & 0.30 & 0.37 \\
\hline & doublet & & & \multicolumn{2}{|c|}{0.55} & \multicolumn{2}{|c|}{0.31} \\
\hline \multirow{2}{*}{1.0} & sextet & 468.0 & 508.0 & 0.02 & 0.08 & 0.30 & 0.37 \\
\hline & doublet & & & \multicolumn{2}{|c|}{0.52} & \multicolumn{2}{|c|}{0.31} \\
\hline
\end{tabular}

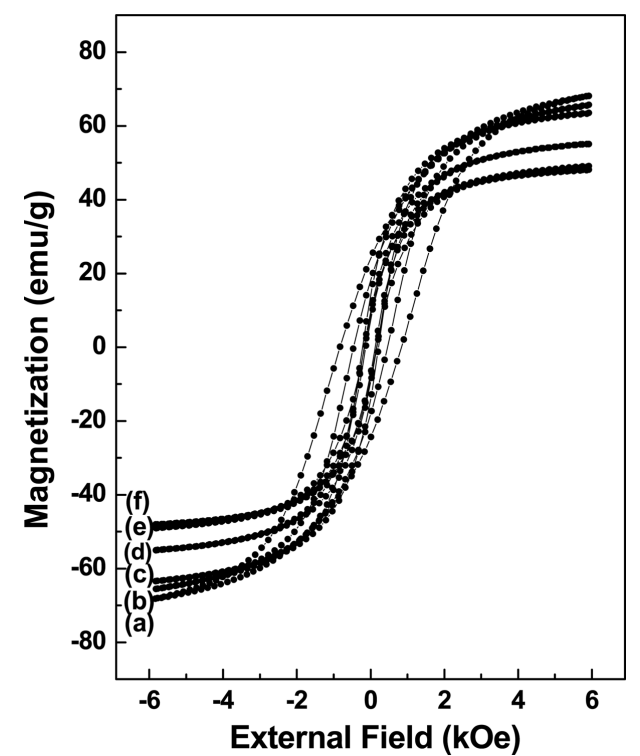

Fig. 5. Hysteresis curves of $\mathrm{CoGa}_{\mathrm{x}} \mathrm{Fe}_{2-\mathrm{x}} \mathrm{O}_{4}$ ferrites: (a) $\mathrm{x}=0.0$, (b) $\mathrm{x}=0.2$, (c) $\mathrm{x}=0.4$, (d) $\mathrm{x}=0.6$, (e) $\mathrm{x}=0.8$ and (f) $\mathrm{x}=1.0$.

the A- and B-site [9] indicate that the cation distribution of our samples is $\left(\mathrm{Co}_{0.2-0.2 \mathrm{x}} \mathrm{Ga}_{\mathrm{x}} \mathrm{Fe}_{0.8-0.6 \mathrm{x}}\right)\left[\mathrm{Co}_{0.8+0.2 \mathrm{x}} \mathrm{Fe}_{1.2-0.4 \mathrm{x}}\right] \mathrm{O}_{4}$. The Fe ions that do not participate in the superexchange process in the spinel structure show doublet in the Mössbauer spectrum. For $\mathrm{x}=0.6$, about $97.0 \%$ of the $\mathrm{Fe}$ ions participate in the superexchange process in this spinel structure. For $\mathrm{x}=1.0$, about $95.0 \%$ of the Fe ions participate do so. That is, the $\mathrm{Fe}^{3+}$ ions that escaped and/ or overflowed from the B-site do not participate in the AO-B superexchange interaction, so they show quadruple splitting in their Mössbauer spectra.

The magnetic properties of the $\mathrm{CoGa}_{\mathrm{x}} \mathrm{Fe}_{2-\mathrm{x}} \mathrm{O}_{4}$ ferrite were determined at room temperature by using a vibrating sample magnetometer, as shown Fig. 5. Fig. 6 and Table 2 show the change in the saturation magnetization $\left(M_{S}\right)$

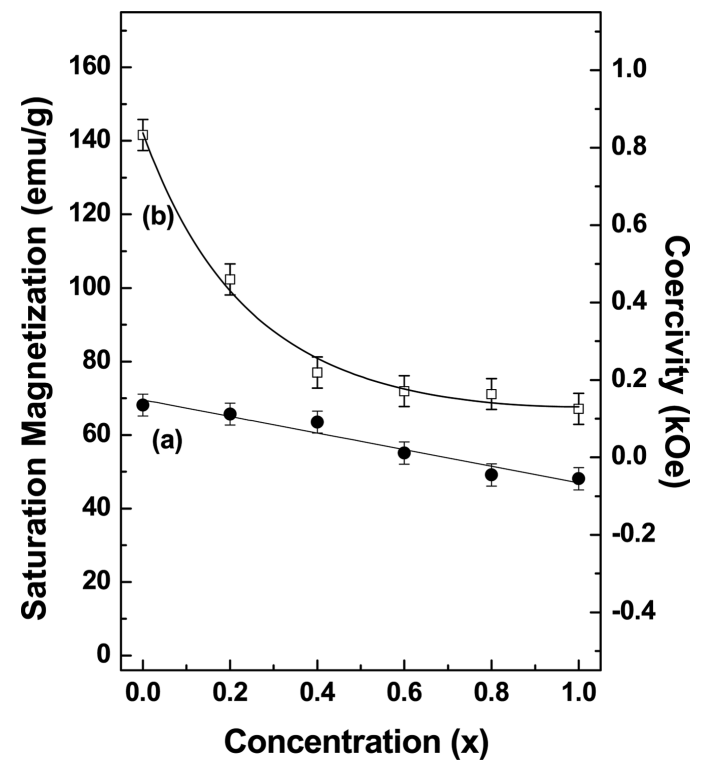

Fig. 6. (a) Saturation magnetization and (b) coercivity of the $\mathrm{CoGa}_{\mathrm{x}} \mathrm{Fe}_{2-\mathrm{x}} \mathrm{O}_{4}$ ferrites.

and the coercivity $\left(H_{C}\right)$ in a maximal field of $6 \mathrm{kOe}$. As the gallium concentration increases, the saturation magnetization decreases and the coercivity abruptly decrease. The maximum saturation magnetization is of $68.1 \mathrm{emu} / \mathrm{g}$ at $\mathrm{x}=0.0$, and the minimum coercivity is 125.4 Oe at $\mathrm{x}=$ 1.0. The variation in the saturation magnetization can be explained through the use of Neel's two sublattice models [10]. The magnetic moment in the inverse ferrites is

Table 2. Room temperature saturation magnetization $\left(M_{s}\right)$ and coercivity $\left(H_{c}\right)$ of $\mathrm{CoGa}_{\mathrm{x}} \mathrm{Fe}_{2-\mathrm{x}} \mathrm{O}_{4}(0.0 \leq \mathrm{x} \leq 1.0)$ ferrite powder.

\begin{tabular}{ccccccc}
\hline \hline $\mathrm{x}$ & 0.0 & 0.2 & 0.4 & 0.6 & 0.8 & 1.0 \\
\hline$M_{s}(\mathrm{emu} / \mathrm{g})$ & 68.1 & 65.7 & 63.5 & 55.1 & 49.1 & 48.1 \\
$H_{c}(\mathrm{Oe})$ & 832.8 & 459.6 & 219.3 & 171.1 & 163.6 & 125.4 \\
\hline
\end{tabular}


mainly a result of the magnetic moments of the A- and Bsite. The A-B superexchange interaction is the predominant interaction, but not in the sublattice A-A and B-B interactions. Hence, the net magnetic moment is given as $\mu=\left(\mu_{B}-\mu_{A}\right)$ since we are substituting the nonmagnetic $\mathrm{Ga}^{3+}$ ion in the A-site, which results in a decrease in the net magnetic moment of the Ga-substituted Co ferrite. The coercivity is known to generally decrease as the particle size decreases as a result of the lower anisotropy barriers [11]. In our samples, the coercivity rapidly decreases, as shown in Fig. 6, which can also be related to the variation in the particle size, as shown in Fig. 2(b). In our case, the variation in the coercivity could be explain as a combination of the effect of the reduced magnetic interaction with the increase in nonmagnetic $\mathrm{Ga}^{3+}$ ions content and the variation in particle size.

\section{Conclusion}

Gallium-substituted cobalt ferrite, $\mathrm{CoGa}_{\mathrm{x}} \mathrm{Fe}_{2-\mathrm{x}} \mathrm{O}_{4}$, was synthesized via conventional ceramic methods, and its crystallographic and magnetic properties were investigated. All of the samples showed a single phase with a spinel structure, the lattice parameters linearly decreased as the substituted Ga content increased, and the particle size of the samples linearly decreased as the value of $x$ increased.

For $\mathrm{x} \leq 0.4$ in $\mathrm{CoGa}_{\mathrm{x}} \mathrm{Fe}_{2-\mathrm{x}} \mathrm{O}_{4}$, the Mössbauer spectra could be fitted with two Zeeman sextets, which is consistent with the typical spinel ferrite spectra of $\mathrm{Fe}^{3+}$ with A- and B-sites. However, for $\mathrm{x} \geq 0.6$ the Mössbauer spectra could be fitted with two Zeeman sextets and one doublet. The variation of the Mössbauer parameters and the absorption area ratio allowed us to determine the cation distribution. The magnetic behavior of the samples showed that an increase in the gallium contents led to a decrease in the saturation magnetization and in the coercivity.

\section{Acknowledgement}

This paper was supported by Konkuk University.

\section{References}

[1] V. Blasko, V. Petkov, V. Rusanov, Ll. M. Martinez, B. Martinez, J. S. Muñoz, and M. Mikhove, J. Magn. Magn. Mater. 162, 331 (1996).

[2] N. N. Greenwood and T. C. Gibb, Mössbauer spectroscopy, Chapman and Hall Ltd. London (1971) p. 261.

[3] M. A. Gabal, S. A. Al-Thabaiti, E. H. El-Mossalamy, and M. Mokhta, Ceramic International 36, 1339 (2010).

[4] Z. K. Heiba, M. B. Mohamed, M. A. Ahmed, M. A. A. Moussa, and H. H. Hamdeh, J. Alloys Compd. 586, 773 (2014).

[5] M. H. Mahmoud, Solid State Ions 176, 1333 (2005).

[6] C. G. Whinfrey, D. W. Eckort, and A. Tauber, J. Am. Chem. Soc. 82, 2695 (1960).

[7] B. D. Cullity, Elements of X-Ray Diffraction, AdditionWesley Co. Readings, MA (1978) p. 102.

[8] K. P. Chae, W. K. Kim, and J. K. Lee, J. Magn. Magn. Mater. 324, 2701 (2012).

[9] W. O. Choi, J. K. Lee, B, S. Kang, and K. P. Chae, J. Magn. 19, 59 (2014).

[10] L. Neel, Ann. Phys. 3, 137 (1948).

[11] C. P. Bean and J. D. Livingston, J. Appl. Phys. 30, 120S (1959) 